\title{
A Indústria Portuguesa na sub-região do Entre Douro e Vouga (EDV): Uma aplicação do ELECTRE III
}

\author{
Portuguese Manufacturing Industry in the sub-region of EDV: An Application of \\ ELECTRE III
}

Antonieta Maria Sousa Lima ${ }^{1}$; Amélia Cristina Ferreira-da-Silva ${ }^{2}$; Eduardo Leite ${ }^{3}$.

1E-mail:

antonieta_sousa_lima@hotmail.com; ISVOUGA

2 E-mail: acfs@iscap.ipp.pt; IPP

3 E-mail:

eduardoleite@gmail.com;

Investigador
Resumo: O propósito deste estudo é construir um ranking das empresas industriais que operam na sub-região do EDV por código de atividade. Assim, utilizamos a metodologia ELECTRE III como ferramenta para construir o rank das empresas e diferenciá-las com bases em rácios financeiros: rentabilidade dos capitais próprios, rentabilidade do ativo, autonomia financeira, e liquidez geral. Estes quatro rácios permitem diferenciar as empresas de acordo com a sua performance quando comparada com as médias do mercado. Estas médias são fornecidas pelas estatísticas do Banco de Portugal. Usando dados de 2010 a 2013, apenas trabalhamos empresas industriais, pois são estas que melhor caracterizam a estrutura de negócios da sub-região. Este estudo baseia-se numa análise descritiva, o que nos permitiu concluir que, da amostra de 185 empresas, após aplicar critérios de exclusão, foi dividida em nove sub-amostras, tendo a maior parte das empresas ficado num dos primeiros três lugares do ranking. Concluímos também que, destas empresas, algumas têm elevados níveis de exportação, estando a maioria em linha com a média do mercado, aqui medida pelas estatísticas do Banco de Portugal.

Palavras-chave: Sub-região do EDV; rácios financeiros; ELECTREIII; empresas industriais; código de atividade (CAE).

\begin{abstract}
The purpose of this study is to construct a ranking of industrial organizations that operate in the sub-region of EDV for ethical code. Thus, we use the methodology ELECTRE III as a tool to build a rank in the industries and differentiate it with financial ratios: return on equity, return on assets, financial autonomy and general liquidity. These four ratios allow to distinguish the organizations based on its performance compared with the market average. This information is provided by the Bank of Portugal. Using data from 2010 to 2013, only focusing on industrial organizations, because these characterize the structure of business in the sub-region. This study uses a descriptive analysis, which permits to conclude that, from the sample of 185 industries, after using the exclusion criteria, it was divided in nine sub-samples, resulting in most of the organizations to belong in one of the three first places in the ranking. We also conclude that, of these companies, there are high levels of exports, the majority being in line with the market's average, here measured by the Statistics of the Bank of Portugal.
\end{abstract}

Palavras-chave: Sub-region of EDV; financial ratios; ELECTRE III; industrial companies; Activity Code (CAE). 


\section{Introdução}

A sub-região do Entre Douro e Vouga (EDV) é uma sub-região que agrega cinco municipalidades, nomeadamente, Santa Maria da Feira, São João da Madeira, Arouca, Vale de Cambra e Oliveira de Azeméis. A estrutura de negócios é baseada, maioritariamente, em atividades industriais, fortemente suportada pelas exportações. Deste modo, as exportações portuguesas são apontadas como um factor-chave para a recuperação económica, assim como para a redução do deficit externo do país. A atividade exportadora é uma importante componente da dinâmica económica, dando o EDV um contributo positivo para as intenções nacionais.

Dadas as características únicas da sub-região do EDV na atividade industrial e exportadora, este artigo estuda as empresas industriais por código de atividade no sentido de as diferenciar pela sua performance financeira. Assim, realizamos um processo step-by-step baseado no método de ranking, ELECTRE III, e um grupo de rácios de 2010 a 2013 relativos a alavancagem, rentabilidade e liquidez, nomeadamente, rentabilidade dos capitais próprios, rentabilidade do ativo, autonomia financeira e liquidez geral. Adicionalmente, para todas as empresas que ficaram num dos primeiros três lugares do ranking, foi também analisada a atividade exportadora.

Nesta sub-região do EDV, as principais indústrias são: madeira e cortiça, couro, metalúrgica, calçado, têxteis, máquinas e equipamentos, produção de acessórios para automóveis, plásticos e borracha. De acordo com Torres (2010), em 2007, esta indústria foi responsável por 52,4\% do emprego, $57 \%$ do total do volume de negócios, e cerca de $63 \%$ do produto interno bruto criado na sub-região.

O artigo está organizado da seguinte forma: em primeiro lugar, é apresentado um enquadramento teórico, mais precisamente, sobre o problema de decisão com base em multi-critérios, as principais características do método ELECTRE III e a importância dos rácios financeiros. Depois, o trabalho empírico é apresentado, seguido dos resultados. Por último, as principais conclusões são discutidas.

\section{Enquadramento Teórico do Modelo de Decisão Multi-Critérios}

Uma decisão é uma escolha entre mais do que uma possibilidade de ação. A palavra decisão pode ser associada a uma série de ferramentas de suporte à decisão, ao processo de decisão ou à teoria de decisão. Consciente ou inconscientemente, as pessoas tomam 


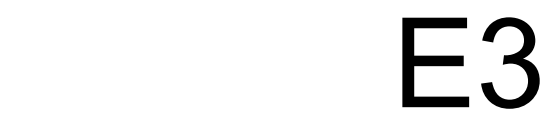

Empresas e Empreendedores

na CPLP

decisões a todo o tempo. Assim, não é de surpreender que o processo de decisão seja muito debatido. Desde a matemática à estatística, através da economia e das ciências políticas, à sociologia e psicologia, cada área de conhecimento desenha a sua perspetiva sobre esta matéria.

Entre os vários processos de decisão, vamos distinguir: (i) decisões planeadas, ou seja, decisões rotineiras e repetitivas, para as quais o decisor já tem uma resposta que aparece de forma rápida e automática. No contexto das organizações, decisões planeadas são delegadas para os níveis mais baixos da hierarquia. (ii) Decisões não planeadas, ou seja, decisões sobre problemas não esperados que requerem uma resposta mais elaborada, incluindo uma análise do problema e a identificação de alternativas. Este tipo de decisão é normalmente tomado nos níveis mais elevados da hierarquia.

Tipicamente, o problema da análise precede a decisão (Kepner \& Benjamin, 1965). Bana, Costa e Vansnick (1997) identificaram que a estruturação e a avaliação são as duas fases principais no processo de decisão, ambas consideradas essenciais na implementação de uma metodologia de suporte ao processo de decisão. A análise do problema envolve a identificação do mesmo e o estudo das suas causas. Um problema poderá ser tanto mais complexo quanto maior for a sua ambiguidade, subjetividade, urgência, dimensão e importância. A decisão requer a definição de objetivos e o estudo de alternativas. Alternativas são sempre ações globais que podem ser implementadas e avaliadas separadamente. O decisor é o responsável por fazer a escolha entre alternativas identificadas como viáveis. Os critérios de decisão são ferramentas que permitem comparar alternativas face aos objetivos.

A abordagem teórica preocupa-se com a natureza da racionalidade e lógica do processo de decisão. A análise descritiva, em contraposição, está preocupada com as preferências das pessoas tal como elas são e não como deveriam ser. A tensão existente entre a perspetiva teórica e a perspetiva descritiva caracteriza muito do estudo de julgamento e escolha (Kahneman \& Tversky, 2000). De facto, o conceito de racionalidade é central no estudo da "teoria da decisão" (Doya \& Shadlen, 2012). O modelo racional descreve o processo de selecionar a melhor alternativa, o melhor rumo da ação de acordo com os valores e objetivos da organização. As escolhas alternativas baseiam-se na informação recolhida, aquela que é considerada como a melhor disponível para resolver os problemas ou atingir os objetivos da organização. As escolhas são indiferentes para os interesses dos participantes. Confrontados com a mesma informação e com o mesmo conhecimento, normalmente, as pessoas chegam a um entendimento. A linguagem 
tecnocrata suporta a "crença" da única e consensual solução, longe da perspetiva pluralista do desentendimento e conflito.

Mas o que é que dita a escolha/decisão? Numa perspetiva racional, é uma análise cuidada da informação, que é a opção que permite maximizar o valor. As decisões são ditadas pela eficiência e eficácia dos critérios.

O método ELECTRE é um bom exemplo de ferramenta de suporte ao processo de decisão numa abordagem racional. O método ELECTRE tem vindo a ser desenvolvido ao longo dos anos. Desde a sua primeira abordagem (Bernard Roy em 1960), foram desenvolvidas várias versões: I, II, III, IV, IS and TRI, e tem sido aplicado a um vasto leque de decisões e áreas (Figueira et al., 2005). Os métodos de ranking são baseados na construção de uma relação que considera preferências do decisor, baseadas nas alternativas disponíveis. Consiste numa família que pertence à área dos Aid Multicriteria Decision Making (AMD), desenvolvida e aplicada pela primeira vez por Bernard Roy, nos anos 60, para resolver um problema de escolha da melhor alternativa a partir de uma série de alternativas, tomando em consideração vários critérios que influenciavam a sua escolha. Esta metodologia foi aplicada para resolver três problemas que envolvem a decisão: a alternativa, a classificação e a escolha (Roy, 1996). Apesar de ter mais de 40 anos de existência, esta abordagem de ranking, em particular o método ELECTRE, continua a ser a abordagem mais popular no campo de pesquisa que envolve MCDA (Govindan \& Jepsen, 2015).

Um processo de decisão, de acordo com Roy (1991), é a representação de um elemento de uma decisão global. Quando se resolve um problema multi-critérios, a dificuldade reside no julgamento de escolhas alternativas sob diversos pontos de vista (EscobarToledo \& López-Garcia, 2005). Zbigniew e Watróbski (2008) definem que um problema de decisão é constituído por dois elementos, $(C, \theta)$, onde $C$ representa um conjunto de critérios, que descrevem as propriedades e níveis de preferência das alternativas consideradas; e $\theta$ representa uma série de dados das expectativas do decisor sobre determinada situação. O elemento fundamental da série de dados $\theta$ é a escolha da problemática, de acordo com o seguinte (Roy, 1991):

- Problema $\alpha$ - um problema de escolha (escolher um subgrupo de A que inclua as melhores soluções); 
- Problema $\beta$ - um problema de seleção (alocar alternativas por categorias);

- Problema $\gamma$-o problema de ordenação ou ranking (construção de um ranking das alternativas, da melhor para a pior).

Tal abordagem considera apenas parte do processo de decisão. Aplicando os métodos multi-critérios, para analisar uma situação de decisão, requer escolher deliberadamente um método que sirva a situação de decisão, por exemplo, o método ELECTRE. O objetivo é encontra uma função, $F(C, \theta)->\max u$, onde $u$ é um indicador da satisfação do decisor medido pelas suas preferências.

\section{Principais características do método Electre}

Os métodos ELECTRE da escola Europeia são considerados métodos relevantes quando se trata de problemas de decisão multi-critérios, tal como indicado por Buchanan, Sheppard e Vanderpooten (1999), Kangas, A., Kangas, J. e Pykäläinen (2001), Figueira, Mousseau e Roy (2005) (seguindo os estudos de Roy (1991), Roy \& Bouyssou (1993), Schärlig (1985), Tervonen et al. (2005), Hanandeh \& El-Zein (2006), Wang (2007), Afshari, Mojahed et al. (2010), e Lima \& Salazar (2011, 2013), entre muitos outros.

A utilização do método ELECTRE baseado em critérios é importante para separar dois tipos de parâmetros: coeficiente de importância e limite de veto. O coeficiente de importância no método ELECTRE refere-se ao peso intrínseco de cada critério: para um dado critério o peso, $w_{j}$, reflete o seu poder de voto quando contribui para a maioria estarem a favor de certo ranking. $O$ limite de veto expressa o poder atribuído a certo critério para estar contra a afirmação " $a$ outranks b", quando a diferença na avaliação entre $g(b)$ e $g(a)$ é maior que este limite. Tais limites podem ser constantes ou variáveis. No método ELECTRE um limite de indiferença $\boldsymbol{q}$, um limite de preferência $\boldsymbol{p}$, e uma relação binária $\boldsymbol{Q}$ são acrescentados. A definição destes limites permite testar todas as alternativas da relação $a S b_{h}$, " $a$ é pelo menos tão bom quanto $b_{h}$ " ou " $a$ não é pior que $b_{h}$ ".

Esta situação dá origem a:

- $\left[a S b_{h}\right.$ e não(b $\left.\left.b_{h} S a\right)\right]: a P b(a$ é estritamente preferido a $b)$;

- [não $\left(a S b_{\mathrm{h}}\right)$ e $\left.b_{\mathrm{h}} S \mathrm{a}\right]: \mathrm{a} \boldsymbol{R b}(a$ é imcomparável a $b)$; 
- $\quad\left[\mathrm{a} S \mathrm{~b}_{\mathrm{h}} \mathrm{e} \mathrm{b}_{\mathrm{h}} \boldsymbol{S a}\right.$ ]: $\mathrm{a} \boldsymbol{l b}(a$ é indiferente a $b$ );

- $\quad\left[\right.$ não $\left(\mathrm{a} S \mathrm{~b}_{\mathrm{h}}\right)$ e não( $\left.\left.\mathrm{b}_{\mathrm{h}} \mathrm{Sa}\right)\right]$ : $\mathrm{a} \boldsymbol{R} \mathrm{b}(a$ é incomparável a $b)$.

No ELECTRE III, a relação de outranking requer a definição de um índice de credibilidade, que caracteriza a afirmação $a \boldsymbol{S} b_{h}$ - "a outranks $b$ " - sendo definida através de um índice de concordância e de um índice de discordância para cada critério $g_{j}$ em $F$.

O índice de concordância $c_{j}(a, b)$ é calculado para cada par de alternativas $(a, b)$ em função de cada critério de decisão. O índice de concordância c $(a, b)$ é a soma dos índices de concordância $\mathrm{c}_{j}(a, b)$ para cada critério devidamente ponderado. Assim:

- Se a performance de $a$ é superior ou igual à de $b$, ou se a performance de $a$ é inferior à de $b$, mas $a$ mantêm-se indiferente a $b$ então $\mathrm{c}_{j}(a, b)=1$;

- Se $b$ é fracamente preferido a $a$ : $\mathrm{c}_{j}(a, b)$ é obtido através de interpolação linear encontramdo-se entre 0 e 1 ;

- Se $b$ é estritamente preferido a $a$ então $\mathrm{c}_{j}(a, b)=0$.

Seguidamente, procedimentos de ordenação descendente e ascendente são aplicados à matriz de credibilidade, no sentido de se construírem duas pré-ordenações das alternativas (Belton \& Stewart, 2001; Rogers et al., 1999). Estando definidas essas duas pré-ordenações, estas são combinadas obtendo-se o ranking final das alternativas.

Dadas as características únicas da família ELECTRE e respetivas versões, o método ELECTRE III foi o escolhido por entrar em linha de conta com limites de preferência e de indiferença, como necessidade de se quantificar a importância de cada critério (ponderação), e por ser específico para problemas de ranking.

\section{A importância da Teoria Financeira}

A teoria financeira e os rácios financeiros, em particular, devem ser considerados para refletir de que forma os dados financeiros podem adicionar conhecimento $\mathrm{e}$ compreensão à forma como algumas empresas deixam de crescer ou entram em insolvência, traduzindo-se no pior pesadelo dos investidores. Infelizmente, a história está cheia de empresas que entraram em insolvência, devido aos mais variados motivos, 


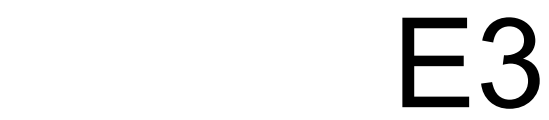

Empresas e Empreendedores

na CPLP

nomeadamente, recessão económica, crise financeira, entre outros. Mas como podem os investidores protegerem-se deste tipo de perdas?

A investigação na previsão de insolvência tem vindo a ser desenvolvida há já muitas décadas, dando origem a um elevado número de publicações desde o estudo pioneiro de Beaver (1966, 1968) e Altman (1968). Beaver (1966) apresentou a evidência empírica de que certos rácios financeiros davam sinais significativos antes de se declarar insolvência. Altman (1968) incrementou o trabalho de Beaver (1966) ao desenvolver a função discriminante que combina os rácios na análise multivariada. Altman (1968, 2000) descobriu que os seus cinco rácios apresentavam uma melhor performance do que os rácios de Beaver (1966). Apesar de cada estudo conseguir discriminar entre empresas saudáveis e empresas com pouca saúde financeira, os vários estudos não concordam nos factores que permitem prever a insolvência.

\section{Metodologia: Objetivos do trabalho empírico e questões de investigação}

O propósito deste estudo é construir um ranking das empresas industriais que operam na sub-região do Entre Douro e Vouga (EDV) por código de atividade (CAE). Através de um processo step-by-step, baseado numa análise descritiva dos resultados obtidos pelo método ELECTRE III, podemos responder às seguintes questões:

- Podem as empresas industriais diferenciarem-se com base na sua performance financeira?

- As empresas que ficam num dos primeiros três lugares têm melhor performance do que a média do mercado? Serão exportadoras?

\section{Metodologia, dados e software usado no trabalho empírico}

Baseado em Spronk e Hallerbach (1997), o nosso trabalho empírico caracteriza-se como um processo step-by-step, onde:

- Primeiro, selecionamos, da base de dados SABI (Bureau van Dijk), todas as empresas registadas na sub-região do EDV entre 2010 e 2013. Este filtro permitiu obter 1.243 empresas. Desta amostra, apenas escolhemos as empresas industriais por CAE. Mais especificamente:

CAE 13 - Fabricação de têxteis;

CAE 15 - Indústria do couro e dos produtos do couro; 
CAE 16 - Indústrias da madeira e da cortiça e suas obras, excepto mobiliário; fabricação de obras de cestaria e de espartaria;

CAE 17 - Fabricação de pasta, de papel, cartão e seus artigos;

CAE 22 - Fabricação de artigos de borracha e de matérias plásticas;

CAE 24 - Indústrias metalúrgicas de base;

CAE 25 - Fabricação de produtos metálicos, excepto máquinas e equipamentos;

CAE 28 - Fabricação de máquinas e de equipamentos, n.e.;

Fabricação de veículos automóveis, reboques, semi-reboques e componentes

CAE 29 - para veículos automóveis.

- Seguidamente, das empresas selecionadas, aplicámos os critérios de exclusão:

- Volume de Negócios <=2,000,000;

- Número de trabalhadores $<10$;

- Volume de Exportação < 500,000 euros;

- Rácios financeiros com valores negativos.

Estes critérios permitiram reduzir a amostra para 185 empresas.

- Em terceiro lugar, separámos a amostra das 185 empresas em várias subamostras, cada uma referênte a um CAE, e, assim, obtivémos nove subamostras.

No sentido de aplicar o método ELECTRE III, definimos Alternativas, Critérios e Limites:

a) Como Alternativas definimos as 185 empresas, divididas nas nove sub-amostras;

b) Como Critérios usámos a teoria financeira, nomeadamente: Rentabilidade dos Ativos (ROA), Rentabilidade dos Capitais Próprios (ROE), Autonomia Financeira (FA), e Liquidez Geral (CL). A média foi calculada de 2010 a 2013, tendo em conta que cada critério apresenta um peso de $25 \%$ no modelo;

c) Como Limites, definimos q (o limite de indiferença) e p (o limite de preferência), para cada critério. Para definir o limite q, usamos as estatísticas do Banco de Portugal por CAE, e o limite $\mathrm{p}$ foi definido com base na yield portuguesa, 10 anos. Sendo o valor dos rácios definidos com base nas regras de 
atribuição de subsídios. Os critérios e respetivos limites encontram-se descritos na Tabela 1.

Aplicando o software ELECTRE III ${ }^{1}$, obtivemos a matriz de ranking. Esta informação apresenta as empresas elencadas, da melhor para a pior, de acordo com os critérios e limites definidos.

Finalmente, baseado na matriz de ranking e conduzindo uma análise descritiva, destacámos as empresas que ficaram num dos primeiros três lugares.

Tabela 1: Limites do modelo

\begin{tabular}{|c|c|c|c|c|c|c|c|c|c|c|c|c|c|c|c|c|c|c|c|c|c|}
\hline \multirow[t]{2}{*}{ DESCRIPTION } & \multicolumn{3}{|c|}{$\begin{array}{c}\text { Thresholds based on government } \\
\text { subsidies criteria attribution }\end{array}$} & \multicolumn{2}{|c|}{ CAE 13} & \multicolumn{2}{|c|}{ CAE 15} & \multicolumn{2}{|c|}{ CAE 16} & \multicolumn{2}{|c|}{ CAE 17} & \multicolumn{2}{|c|}{ CAE 22} & \multicolumn{2}{|c|}{ CAE 24} & \multicolumn{2}{|c|}{ CAE 25} & \multicolumn{2}{|c|}{ CAE 28} & \multicolumn{2}{|c|}{ CAE 29} \\
\hline & $q$ & $p$ & Entity & $q$ & $p$ & $q$ & $p$ & $q$ & $p$ & $q$ & $p$ & $q$ & $p$ & $q$ & $p$ & $q$ & $p$ & $q$ & $p$ & $q$ & $p$ \\
\hline Liquidity & & & & & & & & & & & & & & & & & & & & & \\
\hline Current liquidity (\%) & 1,00 & 1,50 & $\mathrm{POPH}$ & 1,26 & 1,50 & 1,38 & 1,50 & 1,26 & 1,50 & 1,10 & 1,50 & 1,30 & 1,50 & 1,62 & 1,62 & 1,28 & 1,50 & 1,70 & 1,70 & 1,26 & 1,50 \\
\hline Financial structure & & & & & & & & & & & & & & & & & & & & & \\
\hline Financial autonomy $(\%)$ & 25,00 & 30,00 & QREN & 36,34 & 36,34 & 30,97 & 30,97 & 33,51 & 33,51 & 51,55 & 51,55 & 41,504 & 41,50 & 48,50 & 48,50 & 35,01 & 35,01 & 45,79 & 45,79 & 39,57 & 39,57 \\
\hline Profitability & & & & & & & & & & & & & & & & & & & & & \\
\hline $\mathrm{ROE}(\%)$ & 3,00 & 3,50 & Portuguese yild (10 years) & $-2,31$ & 3,00 & 6,33 & 6,33 & $-1,71$ & 3,00 & 12,57 & 12,57 & 19,291 & 19,29 & $-2,43$ & 3,00 & $-0,13$ & 3,00 & 4,98 & 4,98 & 8,94 & 8,94 \\
\hline R0A $(\%)$ & 3,00 & 3,50 & Portuguese yield (10 years) & 4,53 & 4,53 & 8,35 & 8,35 & 4,59 & 4,59 & 12,22 & 12,22 & 17,23 & 17,23 & 3,93 & 3,93 & 5,89 & 5,89 & 8,05 & 8,05 & 11,46 & 11,46 \\
\hline
\end{tabular}

Fonte: Elaboração própria baseada nas estatísticas do Banco de Portugal.

\section{Rácios financeiros aplicados no trabalho empírico}

No sentido de definir quais os rácios financeiros a utilizar para medir a performance financeira de uma empresa, baseámo-nos em Beaver (1966), Altman (1968, 2000), Yap et al. (2010) e, Chen e Shimerda (1981). Estes autores estudaram quais os rácios que melhor prevêem a insolvência de uma empresa e concluíram que não há necessidade de utilizar muitos rácios. Por exemplo, Taffler (1983) começou com oito potenciais rácios e terminou com apenas quarto. De forma similar, no nosso estudo, utilizámos apenas quatro rácios, escolhidos dos existentes na teoria financeira. Esses rácios incidem sobre rentabilidade, alacancagem e liquidez.

A escolha dos rácios baseou-se em dois critérios: na sua popularidade, medida pela frequência de uso, e na boa performance como mostrado nos estudos sobre insolvência. Em particular:

\footnotetext{
${ }^{1}$ De forma a executar o método ELECTRE e a construir a matriz de ranking, usámos o software gentilmente disponibilizado pela Université Paris Dauphine.
} 


$$
\begin{aligned}
& -R O A=\frac{\text { Re sultadoLíquido }}{N} \\
& \text { N Ativo } \\
& - \text { ROE }_{N}=\frac{\text { Re sultadoLíquido }}{N} \\
& -F A=\frac{\text { CapitalPr óprio }_{N}}{\text { Ativo }_{N}} \\
& \text { - } C L_{N}=\frac{\text { Clientes }+ \text { Inventários }+ \text { CaixaeDepósitos }}{N} \\
& \text { PassivoCorrente }_{N}
\end{aligned}
$$

\section{Resultados}

Tal como mencionado, realizámos uma análise descritiva baseada na matriz de ranking obtida com o ELECTRE III. Relativamente ao CAE 13, todas as 8 empresas ficaram em primeiro ou em segundo lugar. Os resultados mostram-nos que a sua performance, medida pelos rácios financeiros, é superior à média do mercado:

$1^{\circ}$ Ranking: $\quad$ - FEPSA-FELTROS PORTUGUESES, S.A.

- CARLOS SOUSA-INDÚSTRIA, LDA.

- CORTADORA NACIONAL DE PÊLO, S.A.

- ARTEFITA-INDÚSTRIA DE PASSAMANARIAS, LDA.

- FERNANDO ALVES DOS SANTOS-INDÚSTRIA DE REV.TÊXTEIS, LDA.

$2^{\circ}$ Ranking: $\quad$ - TRECAR-TECIDOS E REVESTIMENTOS, S.A.

- DUVALLI, S.A.

- ERT TÊXTIL PORTUGAL, S.A.

Destacámos a empresa Fepsa-Feltros Portugueses, S.A., que obteve os melhores rácios $(\mathrm{CL}=3, \quad \mathrm{FA}=49,18 \%, \quad \mathrm{ROA}=7,42 \%$ e $\mathrm{ROE}=14,93 \%)$. Esta empresa é também exportadora, representando $65,43 \%$ do volume de negócios $(6.250 .286,14$ euros de 9.552.317,44 euros). A Cortadora Nacional de Pêlo, S.A. e a Trecar, S.A. também têm interessantes níveis de exportação, cerca de $20 \%$ do volume de negócios. Todas as outras empresas têm um nível de exportação inferior a 10\%. Relativamente aos rácios financeiros, todas as empresas que ficaram em primeiro lugar estão melhores do que a média do mercado, assim como a empresa Trecar, S.A., que ficou em segundo lugar. Todas as outras empresas obtiveram uma performance inferior à média do mercado, exceto para o ROE. 
Para as empresas pertencentes ao CAE 15, apenas três, das 54 empresas selecionadas, ficaram num dos primeiros três lugares:

$1^{\circ}$ Ranking: - VANCAL, LDA.

$2^{\circ}$ Ranking: - SILVA \& COSTA, LDA.

$3^{\circ}$ Ranking: - SÓVIRAS-COMPONENTES

De todas essas empresas, apenas a Vancal, Lda exporta $16,42 \%$ do total do volume de negócios. As restantes exportam menos de $1 \%$ do volume de negócios. Quanto à performance financeira, todas as empresas estiveram melhor do que a média do mercado, como por exemplo, a Vancal, Lda. com uma $\mathrm{CL}=5,13, \mathrm{FA}=83,41 \%$, ROA $=9,29 \%$ e $\mathrm{ROE}=11,11 \%$.

No que diz respeito ao CAE 16, das 39 empresas consideradas, apenas 5 ficaram num dos primeiros três lugares:

$1^{\circ}$ Ranking: - AMÉRICO DE SOUSA \& FILHOS, LDA.

$2^{\circ}$ Ranking: - MANUEL FEIRINHO \& FILHOS, LDA.

$3^{\circ}$ Ranking: - AMORIM \& IRMÃOS, S.A.

- CORTIÇA BENICIA, S.A.

- M.A.SILVA II-CORTIÇAS, LDA.

A empresa Américo de Sousa \& Filhos, Lda. é aquela que apresenta um menor nível de exportação face às outras, todas elas acima dos $20 \%$ do volume de negócios: Amorim \& Irmãos, S.A. com 39,23\% (89.897.555,55 euros de 228.553.191,70 euros), Cortiça Benicia, S.A. com 96,64\% (6.335.352,32 euros de 6.555.547,88 euros) e M.A.Silva II, Lda. com 39,03\% (2.511.925,27 euros de 5.435.948,99 euros). Olhando para os rácios financeiros, todas as empresas obtiveram melhor performance que a média do mercado.

Analisando os resultados atingidos para o CAE 17, verificámos que todas as empresas se encontram num dos primeiros três lugares:

$1^{\circ}$ Ranking: - OLÍMPIO DE OLIVEIRA FONSECA, S.A.

$2^{\circ}$ Ranking: - CARTONAGEM CARDOSO, S.A.

- CARTONEX-ARTIGOS ESCOLARES E DE ESCRITÓRIO, LDA.

$3^{\circ}$ Ranking: - FÁBRICA DE PAPEL E CARTÃO DA ZARRINHA, S.A. 
No entanto, todas as empresas têm um nível de exportação inferior a 1,5\% do volume de negócios. Quanto à performance financeira (em particular os rácios CL e FA), todas as empresas estiveram melhor do que a média do mercado, mas no que diz respeito aos rácios ROA e ROE, estiveram abaixo, como por exemplo, Olímpio de Oliveira Fonseca, S.A. com $C L=5,21, F A=49,05 \%, \mathrm{ROA}=2,51 \%$ e $\mathrm{ROE}=5,03 \%$.

Naturalmente, estes valores devem ser analisados com cuidado, pois a média de mercado é apenas um padrão de comparação.

Os resultados para o CAE 22 indicam que, das 9 empresas, 6 ficaram posicionadas num dos primeiros lugares:

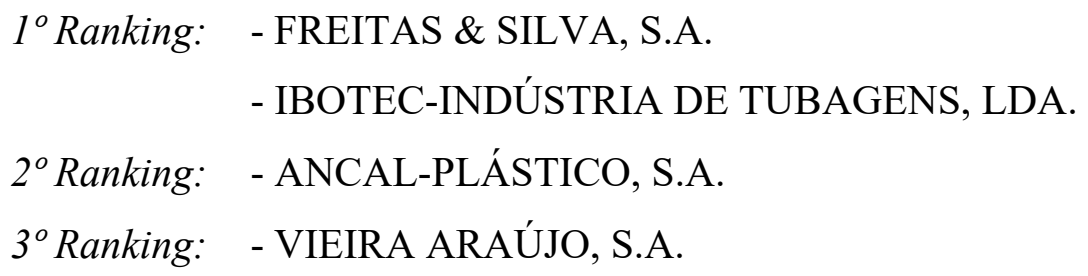

Analisados os níveis de exportação, apenas Freitas \& Silva, S.A. exportou mais de $20 \%$ do volume de negócios. Todas as outras empresas exportaram menos de $2 \%$, exceto a Ibotec, Lda. com um nível de exportação de 8,61\% do volume de negócios. Quanto à performance financeira, uma vez mais, os rácios de rentabilidade (ROA e ROE) ficaram abaixo da média do mercado. De todas as empresas destacámos a Freitas \& Silva, S.A. com $\mathrm{CL}=4,26, \mathrm{FA}=65,97 \%, \mathrm{ROA}=3,21 \%$ e $\mathrm{ROE}=4,91 \%$. Mas, tal como já referido, a média do mercado serve apenas como comparação.

Quanto ao CAE 24, todas as empresas ficam num dos primeiros três lugares:

\author{
$1^{\circ}$ Ranking: - FERPINTA-INDÚSTRIA DE TUBOS DE AÇO DE FERNANDO PINHO \\ - SULIMET, LDA. \\ $2^{\circ}$ Ranking: - ALBERTO DA SILVA BARBOSA \& FILHOS, LDA.
}




\section{- FABRICA VISÃO, LDA.}

Quanto aos níveis de exportação, apenas a Sulimet, Lda. exporta mais de $20 \%$ do volume de negócios, enquanto todas as outras exportam menos de $10 \%$. Analisando os rácios financeiros, destacámos a empresa Ferpinta, Lda. com $\mathrm{CL}=3,80, \mathrm{FA}=75,41 \%$, ROA $=7,45 \%$ e ROE $=10,06 \%$, apesar de apenas exportar $5,05 \%$ do volume de negócios (7.481.287,95 euros de 148.162.097,97 euros). Todas as empresas que ficaram em segundo lugar obtiveram rácios inferiores à média do mercado.

Quanto aos resultados do CAE 25, das 41 empresas, apenas 3 ficaram num dos primeiros três lugares:

$1^{\circ}$ Ranking: - FERBAL-SOCIEDADE FABRIL DE EMBALAGENS, LDA.

$2^{\circ}$ Ranking: - MACAP II-COMÉRCIO E INDÚSTRIA, S.A.

$3^{\circ}$ Ranking: - TETRAMOLD-INDÚSTRIA DE MOLDES, LDA.

Analisados os níveis de exportação, todas as empresas têm níveis abaixo dos $10 \%$ do volume de negócios. No caso particular da empresa Ferbal, Lda., esta empresa exporta apenas $1 \%$. Olhando para a performance financeira, à exceção da empresa Tetramold, Lda., todas as restantes obtiveram melhor performance em relação à média do mercado (Ferbal, Lda. obteve $\mathrm{CL}=7,43, \mathrm{FA}=73,08 \%, \mathrm{ROA}=13,26 \%$ e $\mathrm{ROE}=18,31 \%$ ).

Analisando os resultados do CAE 28, das 10 empresas consideradas, apenas 6 ficaram posicionadas num dos primeiros três lugares:

$1^{\circ}$ Ranking: - GEBO PACKAGING SOLUTIONS PORTUGAL, S.A.

$2^{\circ}$ Ranking: - MARSILINOX-INDÚSTRIA METALÚRGICA, LDA.

- FLUIDOTRÓNICA-EQUIPAMENTOS INDUSTRIAIS, LDA.

$3^{\circ}$ Ranking: - ARSOPI-INDÚSTRIAS METALÚRGICAS ARLINDO S.PINHO, S.A.

- DIVMAC-PROJECTOS AUTOMATISMOS E PERIFÉRICOS IND., S.A.

- AZEVEDOS-INDÚSTRIA, MÁQUINAS E EQUIPAMENTOS IND., S.A.

Quanto aos níveis de exportação, a maior parte apresenta resultados importantes, nomeadamente, Divmac, S.A. com 52,28\% do volume de negócios, Gebo, S.A. com 49,86\% e Arsopi, S.A. com 32,18\%. As restantes apresentam níveis inferiores a 10\%. 
Tendo em conta a performance financeira, todas as empresas obtiveram, pelo menos, um rácio melhor que a média do mercado. Por exemplo, Gebo, S.A. com $\mathrm{CL}=1,66$, $\mathrm{FA}=31,47 \%$, $\mathrm{ROA}=17,07 \%$ e $\mathrm{ROE}=54,42 \%$, valores muito mais elevados que a média do mercado.

Para finalizar, os resultados obtidos para o CAE 29 indicam que todas as 9 empresas ficam num dos primeiros três lugares:

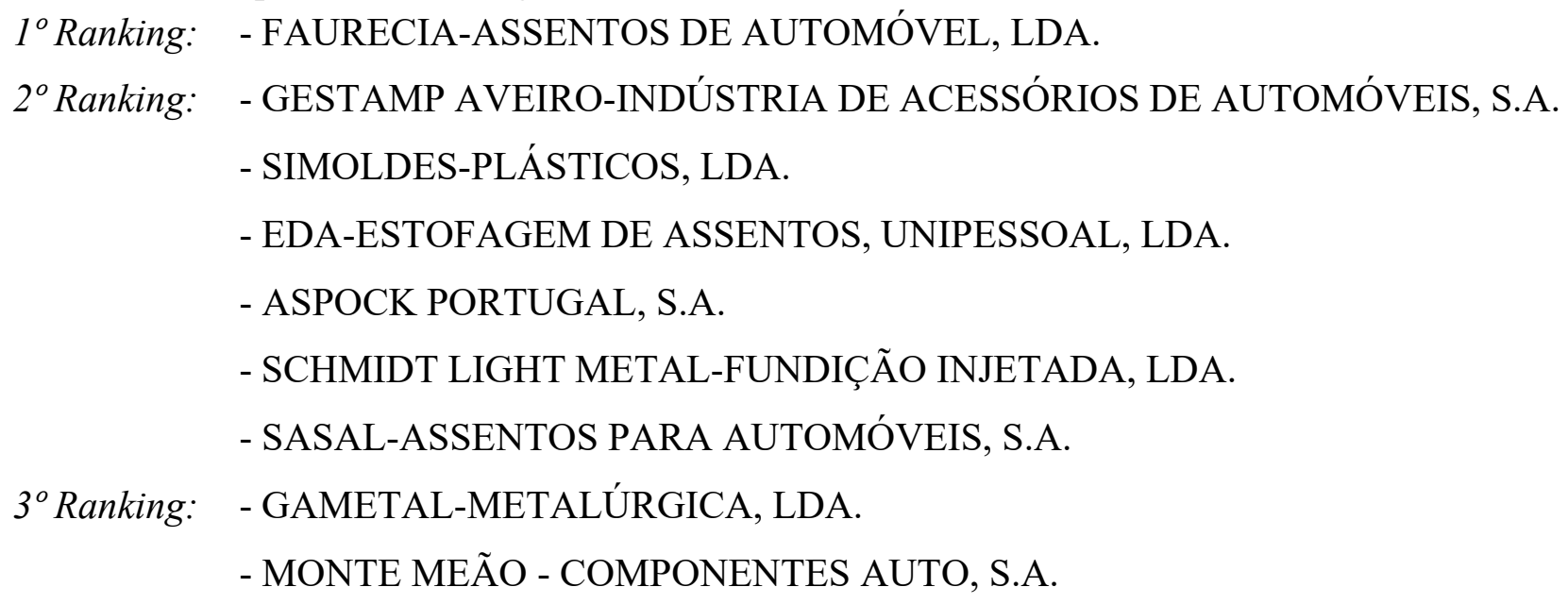

Todas as empresas têm níveis de exportação superiores a 5\% do volume de negócios, exceto a empresa Aspock, S.A. com 9,68\%. Quanto à performance financeira, todas as empresas obtiveram rácios superiores à média do mercado, embora seja de destacar a empresa Faurécia, Lda. com $\mathrm{CL}=0.92, \mathrm{FA}=19,03 \%, \mathrm{ROA}=6,84 \%$ e $\mathrm{ROE}=78,38 \%$.

\section{Conclusões}

Dadas as características únicas da sub-região do EDV, este estudo permitiu realizar um ranking das empresas industriais (da melhor para a pior), por CAE, no sentido de as diferenciar com base na sua performance financeira e nível de exportação, de 2010 a 2013. Neste sentido, conduzimos um processo step-by-step, selecionando um método para realizar o ranking, o ELECTRE III, e definindo um grupo de critério baseados na rentabilidade, alavancagem e liquidez. Mais especificamente escolhemos a ROA, ROE, FA e CL.

De uma amostra inicial de 1.243 empresas, aplicando critérios de exclusão, a amostra foi reduzida para 185 empresas. De seguida, estas 185 empresas foram organizadas pelos CAE mais importantes nesta sub-região, (CAE 13, 15, 16, 17, 22, 24, 25, 28 e 29). Os resultados obtidos com o ELECTRE III indicam que a maior parte das 
empresas ficam num dos primeiros três lugares, sendo que muitas delas apresentaram uma performance financeira superior à média do mercado, medida pelas estatísticas do Banco de Portugal. Estes resultados permitem os seguintes comentários:

- CAE 13 - Fabricação de têxteis: Todas as empresas ficaram num dos primeiros três lugares. Destas empresas apenas a Fepsa-Feltros Portugueses, S.A., Cortadora Nacional de Pêlo, S.A. e Trecar, S.A. exportam mais de $20 \%$ do volume de negócios; as restantes exportam menos de $10 \%$. Quanto à performance financeira, apenas as empresas Duvalli, S.A. e ERT Têxtil Portugal, S.A. ficaram àquem da média do mercado;

- CAE 15 - Indústria do couro e dos produtos do couro: Neste caso, das 54 empresas, apenas 3 ficaram num dos primeiros três lugares. De todas, somente a Vancal, Lda. exporta mais de $10 \%$, mais precisamente, $16,42 \%$; todas as outras exportam menos de 1\%. Quanto aos rácios financeiros, todas as empresas obtiveram melhores resultados em relação à média do mercado;

- CAE 16 - Indústrias da madeira e da cortiça e suas obras, exceto mobiliário; fabricação de obras de cestaria e de espartaria: Das 39 empresas consideradas, apenas 5 ficaram num dos primeiros três lugares.Todas as empresas apresentam melhor performance que a média do mercado e têm níveis de exportação superiores a 20\% do volume de negócios, à exceção de Américo de Sousa \& Filhos, Lda.;

- CAE 17 - Fabricação de pasta, de papel, cartão e seus artigos: Neste caso, todas as empresas selecionadas ficaram num dos primeiros três lugares. Todas as empresas têm níveis de exportação abaixo de $1,5 \%$ do volume de negócios. Quanto à performance financeira e no que respeita aos rácios CL e FA, todas as empresas estiveram melhor que a média do mercado. No entanto, face aos rácios ROA e ROE todas estiveram abaixo;

- CAE 22 - Fabricação de artigos de borracha e de matérias plásticas: Das 9 empresas, apenas 6 ficaram num dos primeiros três lugares. Freitas \& Silva, S.A. é a única empresa que exporta praticamente $20 \%$ do volume de negócios, enquanto todas as outras exportam menos de $2 \%$ (a empresa Ibotec, Lda. exporta $8,61 \%$ do volume de negócios). Uma vez mais, temos empresas com 
uma rentabilidade inferior à média do mercado, em particular no que respeita aos rácios $\mathrm{ROA}$ e ROE;

- CAE 24 - Indústrias metalúrgicas de base: Neste CAE, todas as 4 empresas ficaram num dos primeiros três lugares. De todas, apenas a empresa Sulimet, Lda. exporta mais de $20 \%$ do volume de negócios; as restantes exportam menos de $10 \%$. Olhando para os rácios financeiros, destacamos a empresa Ferpinta, Lda. por ter sido a única que teve melhor performance acima da média do mercado;

- CAE 25 - Fabricação de produtos metálicos, exceto máquinas e equipamentos: Das 41 empresas, apenas 3 ficaram num dos primeiros três lugares. Todas as empresas exportam menos de $10 \%$ do volume de negócios. Quanto à performance financeira, à exceção da empresa Tetramold, Lda., as restantes estiveram melhor do que a média do mercado;

- CAE 28 - Fabricação de máquinas e de equipamentos, n.e.: Olhando para as 10 empresas selecionadas, apenas 6 ficaram num dos primeiros três lugares. Neste caso temos empresas com níveis de exportação muito interessantes, tal como a Divmac, S.A. com 52,28\% do volume de negócios, a Gebo, S.A. com 49,86\% e a Arsopi, S.A. com 32,18\%; as demais têm níveis de exportação inferior a $10 \%$. No que toca à performance financeira, todas as empresas obtiveram pelo menos um rácio melhor do que a média do mercado;

- CAE 29 - Fabricação de veículos automóveis, reboques, semi-reboques e componentes para veículos automóveis: Todas as empresas ficaram num dos primeiros três lugares do ranking. Exceto a empresa Aspock, S.A. que exporta $9,68 \%$ do volume de negócios. Todas as outras exportam menos de 5\%. Quanto à performance financeira, todas as empresas obtiveram rácios superiores à média do mercado, embora seja de destacar a Faurécia, Lda.

Em suma, o método ELECTRE III provou ser uma boa ferramenta de seleção de empresas para investir ou para realizar parcerias, entre outras situações. No entanto, os resultados obtidos apenas deixam em aberto linhas de investigação futuras. Seria proveitoso aplicar outros métodos de multi-critérios, tal como o PROMETHEE, ou enunciar outros pressupostos (critérios e limites). Poderá ser, de igual modo, 
importante caracterizar a sub-região do EDV noutras dimensões (tendo em conta, por exemplo, a estrutura empresarial, o comércio internacional e o nível de exportação), direcionando este estudo para uma possível definição de "elite empresarial".

\section{Bibliografia}

Afshari, R., Mojahed, M., Yusuff, M., Hong, S. and, Ismail, M.Y. (2010). Personnel selection using ELECTRE. Journal Applied Science, 10, 3068-3075. DOI: $10.3923 /$ jas.2010.3068.3075

Altman, E. I. (1968). Financial Ratios, Discriminate Analysis and the Prediction of Corporate Bankruptcy. Journal of Finance, 589-609.

http://www.bus.tu.ac.th/department/thai/download/news/957/Altman_1968.pdf

Altman, E. I. (2000). Predicting Financial Distress of Companies: Revisiting The ZScore and ZETA ${ }^{\circledR}$ Models. http://www.textbiz.org/projects/defaultprediction/zscores.pdf

Beaver, W. H. (1966). Financial Ratios as Predictors of Failure. Journal of Accounting Research, 4, 71111.

Beaver, W. H. (1968). The Information Content of Annual Earnings Announcements. Journal of Accounting Research, 6, 67-92. http://financialaccountingiu.wikispaces.com/file/view/Beaver-1968.pdf

Belton, V. and Stewart, J (2001). Multiple criteria decision analysis: an integrated approach. In Kluwer Academic Publishers, Chapter 8: outranking methods. Boston, MA, USA: Kluwer Academic Publishers.

Buchanan, J., Sheppard, P. and Vanderpooten, D. (1999). Project ranking using ELECTRE III. Paper presented at MCDM. Turkey: Ankara. http://130.217.168.130/departments/staff/jtb/Electwp.pdf

Chen, K. H. and Shimerda T. A. (1981). An Empirical Analysis of Useful Financial Ratios. Financial Management, Spring, 51-60.

Doya, K. and Shadlen, M. (2012). Decision Making. Current Opinion in Neurobiology22 (6): 911-913.

Escobar-Toledo and López-García (2005). The use of multi-criteria decision aid system in the information technology (It) allocation problem. Operational Research, 5(2), 223240 .

Figueira, J., Mousseau, V. and Roy, B. (2005). Multiple Criteria Decision Analysis: State of the Art Surveys. In Springer's International Series (Eds.), Electre Methods (Operations Research and Management Science, 78(III), 133-153). 
http://11.lamsade.dauphine.fr/dea103/ens/bouyssou/Outranking_Mousseau.pdf 
Govindan, K. and Jepsen, M. B. (2015). ELECTRE: A comprehensive literature review on methodologies and applications. European Journal of Operational Research. http://dx.doi.org/10.1016/j.ejor.2015.07.019

Hanandeh, A. and El-Zein, A. (2006). A new stochastic multi-criteria decision analysis tool based on ELECTRE III method. School of Civil Engineering, The University of Sifney.

Kahneman, D. and Tversky, A. (2000). Choice, Values, Frames. The Cambridge University Press. ISBN 0-521-62172-0.

Kangas, A., Kangas, J. and Pykäläinen (2001). Outranking methods as tools in strategic natural resources planning. Silva Fennica, 35(2), 215-227. http://www.metla.fi/silvafennica/full/sf35/sf352215.pdf

Kepner, C.H. and Benjamin B.T. (1965). The rational manager: A systematic approach to problem solving and decision-making. McGraw-Hill

Lima, A. and Salazar, V. (2011). Multi criteria decision making models: An overview on Electre methods. http://wwwa.uportu.pt/siaa/Investigacao/WP_21_2011.pdf

Lima. A. and Soares, V. (2013). Financial Ratios Applied To Portfolio Selection: ELECTRE III methodology in Buy and Hold strategy. ROC, Vol. 9, n 17, pp. 281 a 319. https://www.metodista.br/revistas/revistas-ims/index.php/OC/article/view/281$\underline{319}$

Pfeffer, J. (1981). Power in Organizations. HarperCollins

Picanço, E. (2011). Processo decisório. Universidade Federal Fluminense. Faculdade de Administração, Ciências Contábeis e Turismo. Departamento de Administração.

Rogers, M. G., Bruen, M. and Maystre, L. Y. (1999). Electre and decision support. Chapter 3: the Electre methodology. Boston, MA, USA: Kluwer Academic Publishers.

Rogers, M. G., Bruen, M. and Maystre, L. Y. (1999). Electre and decision support. Chapter 5: case study 1: finding the best location for the galway wastewater treatment plant. Boston, MA, USA: Kluwer.

Rogers, M. G., Bruen, M. and Maystre, L. Y. (1999). Electre and decision support. Chapter 6: case study 2: choosing the best waste incineration strategy for the eastern Switzerland region. Boston, MA, USA: Kluwer Academic Publishers.

Roy, B. and Bouyssou, D. (1993). Aide Multicritère à la Décision: Méthodes et Cas. Édition Économica, Paris.

http://basedpub.dauphine.fr/bitstream/handle/123456789/4522/Couv_Sommaire.pdf?s equence $=1$ 
Roy, B. (1991). The outranking approach and thinks of ELECTRE methods. Theory and Decision, 31 , 
Spronk, J. \& Hallerbach, W. (1997). Financial modelling: Where to go? With an illustration for portfolio management. European Journal of Operational Research, 99, 113-125.

Taffler, R. J. (1983). The Assessment of Company solvency and Performance Using a Statistical Model.

Tervonen, T., Figueira, J., Lahdelma, R. and Salminen, P. (2005). An Inverse Approach for ELECTRE III. In proceedings of the 61th meeting of the EURO EWG, Luxembourg.

Torres, M. L. (2010). Identidade e Dinâmica Socioeconómica da Sub-Região Entre Douro e Vouga. Santa Maria da Feira. Fundação Terras de Santa Maria da Feira.

Wang, X. (2007). Study of ranking irregularities when evaluating alternatives by using some ELECTRE methods and a proposed new MCDM method based on regret and rejoicing (Thesis, Master of Science in Industrial Engineering, Graduate Faculty of the Louisiana State University and Agricultural and Mechanical College, 2007). http://etd.1su.edu/docs/available/etd-07112007-012708/unrestricted/Wang thesis.pdf

Yap, B.C., Yong, D.G. and Poon, W.C. (2010). How Well Do Financial Ratios and Multiple Discriminate

Zbigniew, P. and Watróbski, J. (2008). Environmental factors as determinants of multi-criteria methods suitability for a decision situation. Working paper, Szczecin University of Technology, Faculty of Computer Science and Information Technology, Metody Informatyki Stosowanej. 